\title{
Optical positions of 55 radio stars from astrolabe observations from the Yunnan Observatory
}

\author{
H. Hui and W. Rui \\ Yunnan Observatory, National Astronomical Observatories, Academia Sinica, Kunming 650011, PR China \\ Received 6 July 2001 / Accepted 18 December 2001

\begin{abstract}
The observations by the photoelectric astrolabe at Yunnan Observatory relative to the Hipparcos Catalogue and the optical positions of 55 radio stars were obtained from observations between 1991 and 2000. They all resulted from processing the photon counts obtained by means of the astrolabe after the automation of the instrument. There are 46 stars in common with the Hipparcos Catalogue ${ }^{\star}$.
\end{abstract}

Key words. astrometry - radio continuum: stars

\section{Introduction}

The photoelectric astrolabe of the Yunnan Observatory was installed at the site and put into operation in 1980. The instrument took part in the determination of the Earth Rotation Parameters and joined the Main Campaign of Project MERIT (a program of international collaboration to monitor earth rotation and compare techniques of observation and analysis; Melbourne et al. 1983). Owing to its high observation accuracy the instrument also took part in compiling the General Catalogue of Photoelectric Astrolabes stars (Working group of GCPA 1983) and afterwards in the Chinese Geodetical Stars Catalogue (Working group of CGSC 1991). Upon S. Dèbarbat's (1986) call for intensive observations of radio stars by optical astrometry, 50 radio stars have been selected and observed since 1986. The instrument was automated and equipped with a photon counting detector in the later eighties. It can be operated automatically to observe stars as faint as magnitude 11.0 (Xu et al. 1993). The objects contained in the astrometric catalogue of radio stars by Walter et al. (1990) have all been included in the program to contribute to the linking of the optical reference frame to the VLBI reference frame based on extragalactic objects (Walter \& Sovers 2000). Despite the rainy period lasting nearly half a year in Kunming we succeeded by the end of the year 2000 in deriving the optical positions of 55 radio stars from two transits each

Send offprint requests to: H. Hui,

e-mail: huhui@public.km.yn.cn

* Tables 1 and 2 are also available in electronic form at the CDS via anonymous ftp cdsarc.u-strasbg.fr (130.79.128.5) or via

http://cdsweb.u-strasbg.fr/cgi-bin/qcat?J/A+A/383/1062 between 1991 and 2000. These observations resulted from the data acquired by the photon counting detector after the automation of the instrument.

\section{Observation and reduction}

The radio stars were inserted in the program of 16 basic groups. Each basic group lasts 1.5 hours and involves 40 stars, among which more than 32 stars are related to the FK5 (All FK5 stars are part of the Hipparcos Catalogue). The parameters, $U$ (clock error), $Y$ (latitude correction) and $Z$ (correction of zenith distance for the instrument) are estimated using the FK5 stars in a basic group in which a radio star is inserted. Then the residual is calculated from the following expression:

$V=15 \sin A \cos \varphi_{0}\left(T-T_{0}+U\right)+B_{z}+Y \cos A-Z$

(Dèbarbat \& Guinot 1970; Li et al. 1983), where $T$ is the recorded time of transit and $T_{0}$ is the calculated time of transit of a star. $B_{z}$ accounts for the vacuum correction of the instrument tube and the acceleration correction of the instrument during observation. $\varphi_{0}$ is the adopted latitude of the observing site. $A$ is the transit azimuth of a star, reckoning from north to east, in the range of $0^{\circ}$ to $360^{\circ}$.

Assuming that $V_{\mathrm{e}}$ and $V_{\mathrm{w}}$ are the mean residuals at east and west transits, respectively, the position corrections of the radio stars in right ascension and declination are determined from double transits by the formulas

$\Delta \alpha=\frac{V_{\mathrm{e}}-V_{\mathrm{w}}}{30 \cos \varphi_{0}|\sin A|}$

and

$\Delta \delta=\frac{V_{\mathrm{e}}+V_{\mathrm{w}}-2 K}{2 \cos q}$ 
Table 1. 55 radio star positions (Mean epoch of observations; equator and equinox J2000).

\begin{tabular}{|c|c|c|c|c|c|c|c|c|c|c|}
\hline Name & $\mathrm{RSC}$ & $\mathrm{HIC}$ & Mg. & $\alpha$ & $m_{\alpha}$ & $\delta$ & $m_{\delta}$ & $N_{\mathrm{e}}$ & $N_{\mathrm{w}}$ & $\begin{array}{l}\text { Epoch } \\
-1900\end{array}$ \\
\hline & & & & $\mathrm{h} \mathrm{m} \mathrm{s}$ & $\mathrm{s}$ & $0 \quad 111$ & 11 & & & \\
\hline UU Psc A & 1004 & 1196 & 6.02 & 01458.819 & 0.001 & 84915.48 & 0.02 & 32 & 30 & 96.30 \\
\hline 13 Cet & & 2762 & 5.20 & 03514.786 & 0.002 & -33534.00 & 0.01 & 22 & 50 & 96.51 \\
\hline 39 Cet & 1010 & 5951 & 5.42 & 11636.303 & 0.002 & -23001.14 & 0.01 & 43 & 31 & 96.03 \\
\hline HD 8634 & 1014 & 6669 & 6.18 & 12535.664 & 0.001 & & & 38 & 25 & 93.83 \\
\hline o Cet & & 10826 & 6.47 & 21920.795 & 0.004 & -25838.65 & 0.02 & 38 & 37 & 96.24 \\
\hline 92 Cet & 1025 & 14135 & 2.54 & 30216.773 & 0.001 & 40523.26 & 0.01 & 36 & 35 & 97.78 \\
\hline$\beta$ Per & 1026 & 14576 & 2.09 & 30810.129 & 0.001 & 405720.23 & 0.02 & 25 & 34 & 93.90 \\
\hline UX Ari & 1028 & 16042 & 6.47 & 32635.377 & 0.001 & & & 42 & 36 & 95.58 \\
\hline HR 1099 A & 1029 & 16846 & 5.82 & 33647.292 & 0.001 & 03516.27 & 0.01 & 25 & 38 & 98.08 \\
\hline HD 22403 & 2654 & & 8.30 & 33710.707 & 0.003 & & & 28 & 22 & 94.30 \\
\hline HD 30455 & 1044 & 22349 & 6.95 & 44842.112 & 0.001 & 184234.86 & 0.03 & 47 & 41 & 97.58 \\
\hline$\alpha$ Aur & & 24608 & 0.08 & 51641.335 & 0.002 & 455954.05 & 0.02 & 43 & 56 & 97.06 \\
\hline R Aur & & & 6.50 & 51717.856 & 0.014 & 533509.87 & 0.05 & 39 & 31 & 94.88 \\
\hline$\delta$ Ori A & 2113 & 25930 & 2.25 & 53200.395 & 0.001 & -01756.60 & 0.01 & 68 & 50 & 97.37 \\
\hline$\zeta$ OriA & & 26727 & 1.74 & 54045.517 & 0.002 & -15633.24 & 0.02 & 38 & 32 & 93.91 \\
\hline HD 37806 & 1061 & & 8.00 & 54102.296 & 0.002 & -24300.67 & 0.01 & 31 & 25 & 95.85 \\
\hline 54 Ori & 2123 & 27913 & 4.39 & 55423.012 & 0.001 & & & 49 & 42 & 96.93 \\
\hline$\pi$ Ori & & 28404 & 4.30 & 55956.092 & 0.001 & 455612.25 & 0.01 & 38 & 36 & 95.82 \\
\hline CQ Aur & 2129 & 26715 & 9.04 & 60353.637 & 0.003 & & & 29 & 32 & 94.06 \\
\hline RY Gem & 2166 & & 8.69 & 72724.152 & 0.002 & 153935.21 & 0.08 & 32 & 40 & 93.87 \\
\hline$\sigma$ Gem & 1084 & 37629 & 4.23 & 74318.709 & 0.001 & & & 43 & 33 & 96.72 \\
\hline RZ Cne & 2183 & & 8.67 & 83908.546 & 0.002 & & & 32 & 42 & 93.66 \\
\hline RW UMa & 1118 & & 10.30 & 114046.407 & 0.002 & 515953.48 & 0.02 & 32 & 31 & 93.28 \\
\hline RS CVn & 1137 & 64293 & 8.07 & 131036.926 & 0.001 & & & 34 & 26 & 94.82 \\
\hline $59 \mathrm{Vir}$ & 2256 & 64792 & 5.19 & 131646.620 & 0.001 & 92526.24 & 0.01 & 37 & 21 & 95.69 \\
\hline FK Com & 1140 & 65915 & 8.15 & 133046.813 & 0.004 & & & 34 & 32 & 94.62 \\
\hline $\mathrm{BH} \mathrm{CVn}$ & 1143 & 66257 & 4.91 & 133447.777 & 0.001 & & & 49 & 33 & 95.22 \\
\hline ZZ Boo & 1147 & 68064 & 6.78 & 135609.550 & 0.001 & & & 30 & 29 & 95.78 \\
\hline CU Vir & 2270 & 69389 & 4.99 & 141215.820 & 0.002 & 22434.14 & 0.02 & 32 & 33 & 94.97 \\
\hline 26 Boo & 2608 & 71115 & 5.91 & 143232.578 & 0.001 & & & 45 & 35 & 96.05 \\
\hline 44 Cen & & 73695 & 4.83 & 150347.589 & 0.001 & 473914.43 & 0.01 & 28 & 33 & 93.67 \\
\hline RW CrB & 2298 & & 10.22 & 153915.243 & 0.002 & & & 27 & 30 & 93.35 \\
\hline$\sigma \mathrm{CrB} \mathrm{A}$ & 1172 & 79607 & 5.23 & 161440.958 & 0.002 & & & 38 & 30 & 94.95 \\
\hline U Her & & 80488 & 8.31 & 162547.484 & 0.001 & 185333.12 & 0.03 & 42 & 21 & 93.32 \\
\hline V729 Her & 2340 & 84014 & 8.08 & 171025.609 & 0.004 & 485756.24 & 0.04 & 24 & 25 & 93.30 \\
\hline Z Her & 1189 & 87965 & 7.24 & 175806.994 & 0.001 & 150821.55 & 0.04 & 30 & 28 & 94.75 \\
\hline 59 Ser & 2373 & 90441 & 5.20 & 182712.518 & 0.001 & 01146.10 & 0.01 & 25 & 38 & 96.76 \\
\hline$\beta$ Lyr & 1201 & 92420 & 3.52 & 185004.792 & 0.001 & & & 35 & 30 & 97.10 \\
\hline HD 178208 & 2613 & & 7.60 & 190509.849 & 0.001 & 495523.26 & 0.01 & 42 & 27 & 93.69 \\
\hline HD 179094 & 1207 & 94013 & 5.88 & 190825.832 & 0.003 & 522532.72 & 0.01 & 23 & 39 & 95.66 \\
\hline U Sge A & 2614 & 94910 & 6.50 & 191848.405 & 0.001 & & & 30 & 42 & 96.60 \\
\hline \multirow[t]{2}{*}{$\chi \mathrm{Cyg}$} & & 97629 & 7.91 & 195033.934 & 0.004 & & & 21 & 27 & 93.93 \\
\hline & & 100013 & 6.57 & 201725.181 & 0.001 & 393536.61 & 0.04 & 33 & 35 & 97.55 \\
\hline$\rho \mathrm{Cyg}$ & & 100044 & 4.77 & 201747.203 & 0.001 & & & 31 & 35 & 96.95 \\
\hline V444 Cyg & 2442 & 100214 & 7.93 & 201932.435 & 0.001 & 384353.95 & 0.04 & 21 & 30 & 93.55 \\
\hline V1687 Cyg & 1225 & 100287 & 6.78 & 202027.984 & 0.001 & 435116.10 & 0.02 & 33 & 34 & 97.74 \\
\hline ER Vul & 2460 & 103833 & 7.33 & 210225.893 & 0.001 & & & 29 & 39 & 97.38 \\
\hline HN Peg & 2475 & 107350 & 5.96 & 214431.297 & 0.001 & 144619.39 & 0.02 & 41 & 40 & 97.62 \\
\hline RT Lac & 1246 & 108728 & 8.93 & 220130.716 & 0.003 & 435325.58 & 0.06 & 21 & 23 & 94.08 \\
\hline IM Peg & 1257 & 112997 & 5.86 & 225302.279 & 0.001 & 165028.25 & 0.04 & 31 & 45 & 98.65 \\
\hline RZ And & 2615 & & 10.30 & 230930.043 & 0.017 & 530239.98 & 0.08 & 29 & 27 & 94.83 \\
\hline SZ Psc & 1263 & 114639 & 7.40 & 231323.794 & 0.004 & 24031.66 & 0.04 & 37 & 31 & 95.97 \\
\hline$\lambda$ And & 1265 & 116584 & 3.81 & 233733.748 & 0.001 & 462731.88 & 0.02 & 28 & 34 & 93.77 \\
\hline HD 223460 & 1271 & 117503 & 5.86 & 234940.958 & 0.001 & & & 37 & 41 & 95.87 \\
\hline II Peg & 1272 & 117915 & 7.51 & 235503.862 & 0.002 & & & 21 & 42 & 95.88 \\
\hline
\end{tabular}


Table 2. The external accuracy of the 55 radio star positions.

\begin{tabular}{|c|c|c|c|c|c|c|c|c|c|}
\hline \multirow[b]{2}{*}{ Name } & \multirow[b]{2}{*}{$\mathrm{RSC}$} & \multirow[b]{2}{*}{$\mathrm{HIC}$} & \multirow[b]{2}{*}{$\mathrm{Mg}$. } & \multicolumn{2}{|c|}{ HIC-YPA } & \multicolumn{2}{|c|}{ BORD-YPA } & \multicolumn{2}{|c|}{ USNO-YPA } \\
\hline & & & & $\mathrm{Da}$ & $\mathrm{Db}$ & $\mathrm{Da}$ & $\mathrm{Db}$ & $\mathrm{Da}$ & $\mathrm{Db}$ \\
\hline & & & & & & $\left(0.01^{\prime \prime}\right)$ & & & \\
\hline UU Psc A & 1004 & 1196 & 6.02 & -6 & 7 & -1 & 6 & -3 & 2 \\
\hline 13 Cet & & 2762 & 5.20 & -3 & -12 & & & & \\
\hline 39 Cet & 1010 & 5951 & 5.42 & 17 & 7 & 17 & -13 & 17 & 9 \\
\hline HD 8634 & 1014 & 6669 & 6.18 & -1 & & 2 & & 5 & \\
\hline o Cet & & 10826 & 6.47 & -7 & 3 & & & & \\
\hline 92 Cet & 1025 & 14135 & 2.54 & 1 & -3 & 7 & -5 & & \\
\hline$\beta$ Per & 1026 & 14576 & 2.09 & 2 & 10 & -6 & 9 & & \\
\hline UX Ari & 1028 & 16042 & 6.47 & -3 & & -11 & & -10 & \\
\hline HR 1099 A & 1029 & 16846 & 5.82 & 3 & -3 & 6 & -8 & 4 & 2 \\
\hline HD 22403 & 2654 & & 8.30 & 2 & & 20 & & & \\
\hline HD 30455 & 1044 & 22349 & 6.95 & -8 & 1 & -9 & -4 & -2 & -1 \\
\hline$\alpha$ Aur & & 24608 & 0.08 & 4 & -2 & & & & \\
\hline R Aur & & & 6.50 & -5 & 4 & & & & \\
\hline$\delta$ Ori A & 2113 & 25930 & 2.25 & 8 & -12 & 3 & -17 & & \\
\hline$\zeta$ OriA & & 26727 & 1.74 & 13 & -3 & & & & \\
\hline HD 37806 & 1061 & & 8.00 & -13 & -8 & 4 & -19 & -5 & 1 \\
\hline 54 Ori & 2123 & 27913 & 4.39 & 7 & & 15 & & -2 & \\
\hline$\pi$ Ori & & 28404 & 4.30 & 11 & 2 & 8 & -11 & 4 & 1 \\
\hline CQ Aur & 2129 & 26715 & 9.04 & 19 & & 17 & & 18 & \\
\hline RY Gem & 2166 & & 8.69 & -2 & -21 & & & & \\
\hline$\sigma \mathrm{Gem}$ & 1084 & 37629 & 4.23 & 4 & & -1 & & -4 & \\
\hline RZ Cne & 2183 & & 8.67 & 10 & & -1 & & 0 & \\
\hline RW UMa & 1118 & & 10.30 & 5 & -15 & -17 & -14 & -13 & 6 \\
\hline RS CVn & 1137 & 64293 & 8.07 & 4 & & 4 & & 12 & \\
\hline 59 Vir & 2256 & 64792 & 5.19 & -9 & -9 & -4 & -20 & -6 & -14 \\
\hline FK Com & 1140 & 65915 & 8.15 & 17 & & 23 & & 16 & \\
\hline BH CVn & 1143 & 66257 & 4.91 & -4 & & $\begin{array}{r}-7 \\
\left(0.01^{\prime \prime}\right)\end{array}$ & & -3 & \\
\hline ZZ Boo & 1147 & 68064 & 6.78 & -2 & & 1 & & 9 & \\
\hline CU Vir & 2270 & 69389 & 4.99 & -1 & -4 & 5 & -11 & 5 & -7 \\
\hline 26 Boo & 2608 & 71115 & 5.91 & & & 1 & & 9 & \\
\hline 44 Cen & & 73695 & 4.83 & -18 & 6 & & & & \\
\hline RW CrB & 2298 & & 10.22 & 19 & & -1 & & -17 & \\
\hline$\sigma \mathrm{CrB} \mathrm{A}$ & 1172 & 79607 & 5.23 & 5 & & 3 & & -14 & \\
\hline U Her & & 80488 & 8.31 & -7 & -19 & 7 & -22 & -13 & -18 \\
\hline V729 Her & 2340 & 84014 & 8.08 & -14 & 19 & -17 & 13 & -11 & 10 \\
\hline Z Her & 1189 & 87965 & 7.24 & -7 & -3 & -3 & -7 & -10 & 3 \\
\hline 59 Ser & 2373 & 90441 & 5.20 & 14 & 8 & 13 & -7 & & \\
\hline$\beta$ Lyr & 1201 & 92420 & 3.52 & 4 & & 4 & & & \\
\hline HD 178208 & 2613 & & 7.60 & 15 & 4 & -12 & 13 & -3 & 4 \\
\hline HD 179094 & 1207 & 94013 & 5.88 & 6 & 15 & 8 & 11 & 0 & 21 \\
\hline U Sge A & 2614 & 94910 & 6.50 & 4 & & 6 & & 7 & \\
\hline$\chi \mathrm{Cyg}$ & & 97629 & 7.91 & -1 & & 11 & & -10 & \\
\hline & & 100013 & 6.57 & -6 & 13 & -14 & 22 & & \\
\hline$\rho$ Cyg & & 100044 & 4.77 & & & -1 & & & \\
\hline V444 Cyg & 2442 & 100214 & 7.93 & -17 & 3 & -14 & 6 & & \\
\hline V1687 Cyg & 1225 & 100287 & 6.78 & -10 & 18 & -8 & 17 & -2 & 17 \\
\hline ER Vul & 2460 & 103833 & 7.33 & -4 & & 0 & & 0 & \\
\hline HN Peg & 2475 & 107350 & 5.96 & -6 & -14 & -5 & -17 & 1 & -27 \\
\hline RT Lac & 1246 & 108728 & 8.93 & -10 & -6 & -4 & -7 & & \\
\hline
\end{tabular}


Table 2. continued.

\begin{tabular}{lrrrrrrrrr}
\hline & & & \multicolumn{3}{c}{ HIC-YPA } & \multicolumn{2}{c}{ BORD-YPA } & \multicolumn{2}{c}{ USNO-YPA } \\
Name & RSC & HIC & Mg. & Da & Db & Da & Db & Da & Db \\
\hline IM Peg & 1257 & 112997 & 5.86 & -16 & 8 & -7 & 12 & -12 & 8 \\
RZ And & 2615 & & 10.30 & -3 & -6 & -9 & -13 & -8 & -18 \\
SZ Psc & 1263 & 114639 & 7.40 & -19 & -18 & -12 & -18 & -19 & -16 \\
$\lambda$ And & 1265 & 116584 & 3.81 & -1 & 9 & 6 & -4 & & \\
HD 223460 & 1271 & 117503 & 5.86 & 3 & & 5 & & & \\
II Peg & 1272 & 117915 & 7.51 & 16 & & 23 & & 16 \\
Error & budget, & unit & $0.001^{\prime \prime}:$ & & & & & \\
Mean & difference & & & -4 & 8 & 11 & -40 & -12 & -9 \\
Standard & deviation of & the mean & difference & 14 & 19 & 15 & 25 & 17 & 29 \\
Standard & deviation & of the & difference & 95 & 103 & 101 & 128 & 99 & 127 \\
\hline
\end{tabular}

Here $q$ denotes the parallactic angle. We used the stars with $|\cos q|<0.3$ to calculate $2 K$ from the following expression

$2 K=\frac{\sum P_{i}\left(V_{\mathrm{e}}+V_{\mathrm{w}}\right)_{i}}{\sum P_{i}}$

$P_{i}=-\frac{0.1}{m_{\mathrm{e}}^{2}+m_{\mathrm{w}}^{2}}$

where $m_{\mathrm{e}}$ and $m_{\mathrm{w}}$ are mean errors of $V_{\mathrm{e}}$ and $V_{\mathrm{w}}$, respectively. In this catalogue there are 36 stars, from which one obtains $2 K=0.0045$. Since for $|\cos q|<0.3$ the declinations are obtained by the astrolabe with a rather low precision, they are not given in the catalogue.

Since the Hipparcos Catalogue is the primary realization of the ICRS at optical wavelengths (IAU, 1998), the stellar data of the basic groups have all been adopted from the Hipparcos Catalogue and all the observations have been rereduced since 1986 .

\section{Results}

The optical positions of the 55 radio stars are presented in Table 1 . The mean number of observations of each star is about 33 . The mean precisions are \pm 0.0021 and \pm 0 ". 026 , respectively. The mean epoch of observations is 1995.52 .

To test the external accuracy of the positions given in Table 1 and denoted YPA we made the following three comparisons:

a) HIC-YPA. The difference between the positions of the Hipparcos Catalogue and YPA for the stars in Table 1.

b) BORD-YPA. The positions of stars determined by the Bordeaux meridian circle minus YPA. The Bordeaux results are quoted from the report of Requième \& Mazurier (1991).

c) USNO-YPA. The positions of stars determined by the Flagstaff Astrometric Scanning Transit Telescope of the US Naval Observatory (Stone 1997) minus YPA.

For the comparisons, the positions of stars were reduced to the same epoch by means of the proper motions taken from the Hipparcos Catalogue or from Walter et al. (1990, 1997) before computing differences. The differences are given in Table 2. We also made a statistical analysis of the differences and the results are given in last four rows of Table 2. It can be seen that these quantities all are very small. Therefore YPA's external accuracy is very good.

\section{Explanation of Tables 1 and 2}

Table 1:

Column 1: name of star.

Column 2: the RSC denotes the number in the astrometric catalogue of radio stars.

Column 3: the number in the Hipparcos Catalogue.

Column 4: observed mean visual magnitude.

Columns 5 and \%: right ascension and declination for equator and equinox J2000.0 and epoch of observation.

Columns 6 and 8: mean errors of right ascension and declination.

Columns 9 and 10: the number of the observed transits in east and west, respectively.

Column 11: mean epoch of observations minus 1900.00 .

Table 2:

Column 1: name of star.

Column 2: the RSC denotes the number in the astrometric catalogue of radio stars.

Column 3: the number in the Hipparcos Catalogue.

Column 4: observed mean visual magnitude.

Columns 5 and 6: the differences HIC-YPA in right ascension and declination, respectively. Definition of Da: $\mathrm{Da}=[\alpha(\mathrm{HIC})-\alpha(\mathrm{YPA})] \cos \delta$. The unit of $\mathrm{Da}$ and $\mathrm{Db}$ is $0.01^{\prime \prime}$.

Columns 7 and 8: the differences BORD-YPA in right ascension and declination, respectively. The unit of Da and $\mathrm{Db}$ is $0.01^{\prime \prime}$.

Columns 9 and 10: the differences USNO-YPA in right ascension and declination, respectively. The unit of Da and $\mathrm{Db}$ is $0.01^{\prime \prime}$.

Acknowledgements. The authors heartily thank Professor H. G. Walter and Professor Li Dongming for their valuable help. This work is supported by the Chinese Astronomic Committee Foundation. 


\section{References}

Dèbarnat, S. 1986, IAU WG Commission 24 letter

Dèbarbat, S., \& Guinot, B. 1970, La Méthode des hauteurs égales en Astronomie, Paris

IAU 1998, IAU Inf. Bull., 81, 30

Li Dongming, Xu Jiayan, \& Luo Dingjiaing 1983, Equal altitude method and its application to fundamental astrometry (Science Press, Bejing, in Chinese)

Melbourne, W., Anderle, R., Feissel, M., et al. 1983, Project MERIT standards, USNO Circ, 167

Requième, Y., \& Mazurier, J. M. 1991, A\&AS, 89, 311

Stone, R. C. 1997, AJ, 114, 1679
Walter, H. G., Hering, R., \& de Vegt, Ch. 1990, A\&AS, 86, 357

Walter, H. G., Hering, R., \& de Vegt, Ch. 1997, A\&AS, 122 , 529

Walter, H. G., \& Sovers, O. J. 2000, Astrometry of fundamental catalogues (Springer, Berlin-Heidelberg-New York)

Working group of GCPA, 1983, The General Catalogue of Stars with System of Photoelectric Astrolabes in China, Acta Astron. Sin., Vol. 24 (in Chinese)

Working group of CGSC, 1991, The Chinese Geodetical Stars Catalogue, Mapping Press, Bejing (in Chinese)

Xu Jiayan, Wang Hongqis, Li Dongming, et al. 1993, A\&A, 271,360 